\title{
Perfil clínico del adulto mayor atendido ambulatoriamente en un hospital general.
}

\author{
Clinical profile of elder patients attending an outpatient setting of a general hospital. \\ Paola Casas ${ }^{1}$, Luis Varela², Tania Tello³, Pedro Ortiz ${ }^{4}$, Helver Chávez ${ }^{2}$.
}

\section{RESUMEN}

Objetivo: Describir el perfil clínico del adulto mayor atendido ambulatoriamente en un hospital general. Material y métodos: Estudio descriptivo, transversal, realizado a través de encuestas aplicadas a pacientes en la consulta ambulatoria de geriatría del Hospital Nacional Cayetano Heredia ( $\mathrm{HNCH})$, entre agosto de 2011 y enero 2012. Resultados: Se evaluaron 290 pacientes, el 69,3\% fueron mujeres; el 65,5\% tenía entre 60 y 79 años. La hipertensión arterial fue el diagnóstico más frecuente (55,5\%). El 83,1\% consumía algún tipo de medicamento y el $45 \%$ más de 3 fármacos. En la valoración por esferas: el 83,5\% era autónomo, el 18,1\% tenía deterioro cognitivo, el 7,7\% depresión, el 79,3\% riesgo social, el 23,8\% disfunción familiar, el 31,4\% riesgo de malnutrición, el 23,4\% obesidad, el 96\% mala higiene del sueño y el $4,9 \%$ riesgo de caídas. El síndrome geriátrico más frecuente fue caídas en $23,8 \%$ y de los problemas geriátricos, el edentulismo en 77,6\%; 17,4\% eran frágiles según la velocidad de la marcha; se encontró asociación entre fragilidad y edad avanzada, género femenino y la presencia de riesgo social. Conclusión: Los pacientes que acuden a la consulta externa de Geriatría fueron mayoritariamente mujeres, funcionalmente autónomos, sin deterioro cognitivo, con riesgo social y adecuada funcionalidad familiar, prefrágiles y con enfermedades crónicas no transmisibles principalmente.

PALABRAS CLAVE: Ancianos, signos y síntomas, atención ambulatoria. (Fuente: DeCS BIREME)

\section{SUMMARY}

Objective: To describe the clinical profile of elder patients attending an outpatient setting of a general hospital. Methods: Descriptive and cross-sectional study performed using surveys applied to patients attending Hospital Nacional Cayetano Heredia's elder outpatient clinic between August 2011 and January 2012. Results: 290 patients were evaluated; $69.3 \%$ were females; $65.5 \%$ had $60-79$ years old. Blood hypertension was the most frequent diagnosis in $55.5 \%$. Use of at least one drug was found in $83.1 \%$ and $45 \%$ used more than three drugs; $83.5 \%$ were autonomous; $18.1 \%$ had cognitive deterioration; $7.7 \%$ had depression; $79.3 \%$ had social risk; $23.8 \%$ had dysfunctional families; $31.4 \%$ were at risk of malnutrition; $23.4 \%$ had obesity and $4.9 \%$ had risk of falls. Elderly falls was the most frequent geriatric syndrome $(23.8 \%)$ and edentulism was the most frequent geriatric problem (77.6\%); $17.4 \%$ were fragile based on walking speed; an association was found between fragility, advanced age,

Médico Geriatra, Hospital Nacional Cayetano Heredia. Lima, Perú.

Médico Internista-Geriatra, Hospital Nacional Cayetano Heredia. Instituto de Gerontología, Universidad Peruana Cayetano Heredia. Lima, Perú.

3 Médico Geriatra, Hospital Nacional Cayetano Heredia. Instituto de Gerontología, Universidad Peruana Cayetano Heredia. Lima, Perú.

4 Médico Internista, Hospital Nacional Arzobispo Loayza. Instituto de Gerontología, Universidad Peruana Cayetano Heredia. Lima, Perú. 
female gender and social risk. Conclusion: The majority of elder patients attending the outpatient setting were functionally autonomous females, with no cognitive deterioration, adequate family functioning, pre-fragile and with chronic non-transmissible diseases.

KEY WORDS: Aged, signs and symptoms, Ambulatory care. (Source: MeSH NLM)

\section{INTRODUCCIÓN}

El envejecimiento poblacional, proceso heterogéneo y acelerado a nivel mundial no excluye a nuestro país que según el último censo nacional 2007 (1), proporcionalmente este grupo etario se viene incrementando progresivamente: 5,9\% en 1972 a $9,1 \%$ en 2007. Además, se acompaña de una transición epidemiológica, en relación al tipo de enfermedades prevalentes. Según el estudio de carga de enfermedad del Ministerio de Salud las enfermedades no transmisibles generan la mayor cantidad de años de vida saludables perdidos (2).

Ante esta realidad, se hace necesario el uso de una valoración geriátrica integral que permita realizar intervenciones adecuadas y pertinentes. En Perú, esto se propone por primera vez en el año 1999, en que Varela y col (3), incorporan a la historia clínica convencional la evaluación de las esferas funcional, afectiva, cognitiva y social y la búsqueda de problemas y síndromes geriátricos.

En el Hospital Nacional Cayetano Heredia, las atenciones en la consulta externa de Geriatría han variado de 4,4 a $5,7 \%$ entre el 2008 y el 2011 en el departamento de Medicina (4).

Al no haber información sistematizada de la población adulta mayor que acude a la consulta externa de Geriatría, se realizó el estudio con el objetivo de describir el perfil clínico del adulto mayor atendido ambulatoriamente.

\section{MATERIAL Y MÉTODOS}

Estudio descriptivo, transversal realizado en pacientes del consultorio externo de geriatría del Hospital Nacional Cayetano Heredia entre el 1 de agosto 2011 y el 30 de enero 2012.

La muestra se tomó en base a una representatividad del $50 \%$ de las atenciones brindadas en el consultorio en el periodo de tiempo inmediato anterior similar al del estudio (agosto 2010 a enero 2011) realizándose una aleatorización sistemática con una fracción de muestreo de 2. Se incluyeron a todos los pacientes aleatoriamente seleccionados mayores de 60 años y se excluyeron a los que se negaron a firmar el consentimiento informado.

La recopilación de los datos se realizó en una ficha elaborada por la investigadora. Se tomaron datos de la historia clínica, sociodemográficos, peso, talla, condición de nuevo o continuador, antecedentes patológicos personales, uso de fármacos y diagnósticos del médico tratante al momento de la evaluación. Además, se evaluó al paciente en las siguientes áreas: funcional con el índice de Katz (5), cognitiva con el test de Pfeiffer (6), afectiva con el test abreviado GDS-4 (7), social con la Escala de Gijón (8), funcionalidad familiar con el test de APGAR (9), riesgo de malnutrición con el "Mini Nutritional

\begin{tabular}{|c|c|c|c|}
\hline & MUJERES (\%) & VARONES (\%) & Total (\%) \\
\hline \multicolumn{4}{|l|}{ PROCEDENCIA } \\
\hline Cono Norte & $147(50,6)$ & $71(24,6)$ & $218(75,2)$ \\
\hline Otros distritos & $39(13,5)$ & $4(1,3)$ & $43(14,8)$ \\
\hline Otras ciudades & $15(5,2)$ & $14(4,8)$ & $29(10,0)$ \\
\hline Total & $201(69,3)$ & $89(30,7)$ & \\
\hline \multicolumn{4}{|l|}{ ESTADO CIVIL } \\
\hline Soltero & $17(5,8)$ & $8(2,8)$ & $25(8,6)$ \\
\hline Casado & $69(23,8)$ & $62(21,6)$ & $131(45,4)$ \\
\hline Divorciado & $5(1,7)$ & $3(0,9)$ & $8(2,6)$ \\
\hline Viudo & $110(37,9)$ & $16(5,5)$ & $126(43,4)$ \\
\hline Total & $201(69,3)$ & $89(30,7)$ & \\
\hline \multicolumn{4}{|c|}{ GRADO DE INSTRUCCIÓN } \\
\hline Analfabeto & $39(13,5)$ & $6(2,0)$ & $45(15,5)$ \\
\hline Primaria incompleta & $75(25,8)$ & $14(4,8)$ & $89(30,6)$ \\
\hline Primaria completa & $47(16,2)$ & $36(12,4)$ & $83(28,6)$ \\
\hline Secundaria incompleta & $15(5,2)$ & $8(2,8)$ & $23(8,0)$ \\
\hline Secundaria completa & $16(5,5)$ & $13(4,5)$ & $29(10,0)$ \\
\hline Superior incompleta & $3(0,9)$ & $1(0,6)$ & $4(1,5)$ \\
\hline Superior completa & $6(2,0)$ & $11(3,9)$ & $17(5,9)$ \\
\hline Total & $201(69,3)$ & $89(30,7)$ & \\
\hline
\end{tabular}


Perfil clínico del adulto mayor atendido ambulatoriamente en un hospital general.

Tabla 2. Valoración funcional, cognitiva y afectiva de adultos mayores atendidos en el consultorio externo de Geriatría de acuerdo a género.

\begin{tabular}{|c|c|c|c|}
\hline & MUJERES (\%) & VARONES (\%) & Total (\%) \\
\hline \multicolumn{4}{|c|}{ VALORACIÓN FUNCIONAL } \\
\hline Dependiente & $38(13,1)$ & $10(3,4)$ & $48(16,5)$ \\
\hline Independiente & $163(56,2)$ & $79(27,3)$ & $242(83,5)$ \\
\hline Total & $201(69,3)$ & $89(30,7)$ & 290 \\
\hline \multicolumn{4}{|c|}{ VALORACIÓN COGNITIVA } \\
\hline Con Deterioro & $40(13,9)$ & $12(4,2)$ & $52(18,1)$ \\
\hline Sin Deterioro & $158(52,4)$ & $76(29,5)$ & $234(81,9)$ \\
\hline Total & $198(76,3)$ & $88(33,7)$ & 286 \\
\hline \multicolumn{4}{|c|}{ VALORACIÓN AFECTIVA } \\
\hline Depresión & $16(5,9)$ & $5(1,8)$ & $21(7,7)$ \\
\hline Riesgo de depresión & $5(1,8)$ & $3(1,3)$ & $8(3,1)$ \\
\hline No depresión & $168(62,2)$ & $73(27,0)$ & $241(89,2)$ \\
\hline Total & $189(69,9)$ & $81(30,1)$ & 270 \\
\hline \multicolumn{4}{|l|}{ VALORACIÓN SOCIAL } \\
\hline Problema Social & $5(1,7)$ & $2(0,8)$ & $7(2,5)$ \\
\hline Riesgo Social & $158(54,4)$ & $72(24,9)$ & $230(79,3)$ \\
\hline No riesgo Social & $38(13,1)$ & $15(5,1)$ & $53(18,2)$ \\
\hline Total & $201(69,3)$ & $89(30,7)$ & 290 \\
\hline \multicolumn{4}{|l|}{ DISFUNCIÓN FAMILIAR } \\
\hline Disfunción Familiar grave & $13(4,6)$ & $7(2,6)$ & $20(7,2)$ \\
\hline Disfunción Familiar leve & $27(9,7)$ & $19(6,9)$ & $46(16,6)$ \\
\hline No disfunción familiar & $154(55,5)$ & $57(20,5)$ & $211(76,1)$ \\
\hline Total & $194(70,0)$ & $83(30,0)$ & 277 \\
\hline
\end{tabular}

Assessment"(MNA) (versión acortada) (10), fragilidad usando como marcador la velocidad de la marcha (11), riesgo de caídas con el test de "Get up and Go" (12) e higiene del sueño con el índice de higiene del sueño modificado (13).

Los datos obtenidos fueron procesados en el programa SPSS 13 previa codificación. Se utilizó estadística descriptiva, frecuencias y porcentajes para las variables categóricas y medias y desviación estándar para las numéricas continuas. Se buscó asociación entre variables mediante Chi cuadrado y ANOVA. Se consideró significativo un $\mathrm{p}<0.05$.

\section{RESULTADOS}

Se evaluaron 290 pacientes: 201 (69,3\%) fueron mujeres; $45,1 \%$ fueron nuevos. La edad promedio fue de 76,3 $\pm 8,9$ años (rango: 60 a 100); 190 (65,5\%) tenían entre 60 a 79 años y $100(34,5 \%)$ fueron mayores de 80 años. La procedencia, estado civil, y grado de instrucción se muestran en la tabla 1.
Tabla 3. Síndromes y problemas geriátricos de adultos mayores atendidos en el consultorio externo de Geriatría de acuerdo a género.

\begin{tabular}{lccc}
\hline $\begin{array}{l}\text { SíNDROMES } \\
\text { GERIÁTRICOS }\end{array}$ & MUJERES & VARONES & Total (\%) \\
\hline Caídas & 54 & 15 & $69(23,8)$ \\
Deterioro cognitivo & 40 & 12 & $52(18,1)$ \\
Incontinencia & 31 & 14 & $45(15,5)$ \\
Inmovilismo & 16 & 9 & $25(8.6)$ \\
Delirio & 2 & 1 & $3(1,0)$ \\
PROBLEMAS & & & \\
GERIÁTRICOS & 166 & 59 & $225(77,6)$ \\
Edentulismo & 113 & 39 & $152(52,4)$ \\
Deprivación visual & 86 & 37 & $123(42,4)$ \\
Deprivación auditiva & 40 & 29 & $69(23,8)$ \\
\hline Estreñimiento & &
\end{tabular}

En antecedentes patológicos, 52,1\% tenía hipertensión, $24,5 \%$ osteoartrosis, $23,4 \%$ osteoporosis y $10 \%$ diabetes. El $83,1 \%$ recibía algún tipo de medicamento, siendo los grupos más frecuentes: antihipertensivos $(51,1 \%)$, analgésicos $(28,4 \%)$, antitrombóticos $(25,2 \%)$, vitaminas $(23,8 \%)$, calcio $(20,7 \%)$, y vacunas $(16,8 \%)$. El $45,0 \%$ consumía tres o más medicamentos (polifarmacia), con distribución similar entre mujeres y varones.

En la tabla 2, se muestran los resultados de las valoraciones funcional, cognitiva, afectiva y social, y la funcionalidad familiar. El 16,5\% tenía algún grado de dependencia, con presencia de cuidador en 26 (9\%), $18,1 \%$ algún grado de deterioro cognitivo, $79,3 \%$ tenía riesgo social y $23,8 \%$ disfunción familiar.

El 31,4\% tenían riesgo de malnutrición (según MNA) y $23,4 \%$ tenía obesidad. El síndrome geriátrico más frecuente fue caídas: $23,8 \%$; además, el 77,6\% tenía edentulismo (Tabla 3).

La fragilidad se evaluó por medio de la velocidad de la marcha; la media fue de $0,92 \pm 0,23 \mathrm{~m} / \mathrm{s}$ (rango: 0,23 - 1,58). La distribución de acuerdo a fragilidad, edad y género se muestran en la tabla 4. Se encontró asociación significativa entre fragilidad y edad $\geq 80$ años; entre fragilidad y género femenino $(p<0,001)$ y entre fragilidad y riesgo social: $17,9 \%$ en pacientes sin riesgo social en comparación con $82,1 \%$ en pacientes con riesgo social $(p<0,001)$.

Con el Test "Get up and go" (punto de corte $\geq 20$ seg), el 4,9\% tenía riesgo de caídas. El 96\% tenía mala higiene del sueño (valores por debajo del percentil $95)$, sólo un paciente $(0,35 \%)$ cumplió con todas las prácticas para una buena higiene del sueño. 
Tabla 4. Fragilidad de adultos mayores atendidos en el consultorio externo de Geriatría de acuerdo a género y edad.

\begin{tabular}{lrrrrr}
\hline & \multicolumn{2}{c}{ GÉNERO } & \multicolumn{2}{c}{ EDAD } & Total (\%) \\
\cline { 2 - 5 } & Mujeres (\%) & Varones (\%) & $<\mathbf{8 0 ~ a ~ ( \% ) ~}$ & $\mathbf{2 8 0}$ a (\%) & \\
\hline Frágiles & $32(13,7)$ & $7(3,7)$ & $14(6,2)$ & $25(11,2)$ & $39(17,4)$ \\
Pre-frágiles & $100(44,8)$ & $38(17,0)$ & $110(49,3)$ & $28(12,5)$ & $138(61,8)$ \\
Robustos & $26(11,6)$ & $20(9,2)$ & $42(18,8)$ & $4(2,0)$ & $46(20,8)$ \\
Total & $158(70,1)$ & $65(29,9)$ & $166(74,3)$ & $57(25,7)$ & 223 \\
\hline
\end{tabular}

Tabla 5. Diagnósticos más frecuentes en adultos mayores por órganos y sistemas.

\begin{tabular}{|c|c|c|}
\hline & $\mathbf{n}$ & $\%$ \\
\hline SISTEMA CARDIOVASCULAR & 206 & 71,0 \\
\hline Hipertensión arterial & 161 & 55,5 \\
\hline Dislipidemia & 19 & 6,5 \\
\hline Fibrilación auricular & 17 & 5,8 \\
\hline SISTEMA OSTEOARTICULAR & 182 & 62,7 \\
\hline Osteoartrosis & 78 & 26,8 \\
\hline Osteoporosis & 74 & 25,5 \\
\hline Lumbalgia & 21 & 21,0 \\
\hline SISTEMA GASTROINTESTINAL & 117 & 40,3 \\
\hline Estreñimiento & 69 & 23,7 \\
\hline Dispepsia & 21 & 7,2 \\
\hline Dolor abdominal & 15 & 5,1 \\
\hline SISTEMA ENDOCRINO & 57 & 19,6 \\
\hline Diabetes & 45 & 15,5 \\
\hline Hipotiroidismo & 9 & 3,1 \\
\hline SISTEMA RESPIRATORIO & 54 & 18,6 \\
\hline Enfermedad pulmonar crónica & 45 & 15,5 \\
\hline Rinofaringitis & 9 & 3,1 \\
\hline $\begin{array}{l}\text { SISTEMA NEUROLÓGICO Y } \\
\text { TRAST. PSIQUIÁTRICOS }\end{array}$ & 40 & 13,7 \\
\hline Demencia & 13 & 4,4 \\
\hline Síndrome ansioso-depresivo & 10 & 3,4 \\
\hline Parkinsonismo & 8 & 2,7 \\
\hline TOTAL & 290 & 100,0 \\
\hline
\end{tabular}

Los diagnósticos de los pacientes evaluados se muestran en la tabla 5. El diagnóstico más frecuente fue hipertensión arterial.

\section{DISCUSIÓN}

La población evaluada fue mayoritariamente de género femenino, con un rango predominante de edad entre 60 a 79 años, lo que es concordante con el perfil del envejecimiento poblacional en los diferentes países demostrado en los estudios a nivel nacional como el INTRA III y a nivel latinoamericano como el estudio de salud, bienestar y envejecimiento (SABE) $(14,15)$.

El diagnóstico más frecuente de consulta fue hipertensión arterial $(55,5 \%)$, similar a la reportada por el estudio Tornasol en 2006 (16) en el que encontraron $48,1 \%$ con HTA en adultos mayores de 60 años de la comunidad, probablemente en relación a que la edad es un factor de riesgo cardiovascular importante en este grupo etario.

El estudio de carga de enfermedad (2), atribuye $58 \%$ de las causas de ésta a enfermedades no transmisibles como las neuropsiquiátricas, lesiones no intencionales (caídas), cardiovasculares, neoplásicas y osteoarticulares. Miranda y col (17) al evaluar el perfil de mortalidad en nuestro país considerando la transición demográfica entre los años 1996 y 2000, encontró una transición de la mortalidad por causas transmisibles a no transmisibles, atribuyéndose a éstas últimas más de la mitad de causas de mortalidad. Nuestro estudio encontró un perfil similar siendo las enfermedades cardiovasculares, osteoarticulares, endocrinológicas y respiratorias las más frecuentes.

En nuestro estudio, 83,1\% usaba algún tipo de medicamento, cifra similar a otros reportes nacionales e internacionales. Secoli y col (18) en Reino Unido, encontró que $2 / 3$ de los adultos mayores usaban medicamentos prescritos, en EEUU $1 / 3$ de las prescripciones médicas eran para adultos mayores, en Brasil 84,3\% de los adultos mayores usaban medicación y Ocampo y col (19) en Colombia, encontró que $71,8 \%$ de adultos mayores consumían algún tipo de medicamento.

La OMS considera polifarmacia al uso de 3 ó más medicamentos y se reporta que entre el 65 a $94 \%$ de los adultos mayores consumen algún tipo de fármaco. En nuestro estudio se encontró polifarmacia en 45\%.

El $16,5 \%$ tuvo algún grado de dependencia funcional principalmente en mujeres, lo que es similar a otros estudios realizados en la comunidad. Pinheiro y col (20) encuentra que el género no sería un factor de riesgo para desarrollar dependencia funcional sino 
la edad y las condiciones de salud, socioeconómicas y sociales de la persona.

El $18,1 \%$ tuvo deterioro cognitivo y $4,9 \%$ deterioro severo, datos similares a los presentados por el estudio de Salud Mental en el 2002 (21) en el que sólo en Lima se encontró deterioro cognitivo en $10,5 \%$ de la población evaluada. Se deben realizar estudios que no sólo confirmen esta frecuencia sino también la etiología.

El test abreviado GDS-4 fue validado en nuestro país, teniendo una sensibilidad de $93 \%$ y un valor predictivo negativo de $98 \%$ (7). Utilizando este test se encontró 7,7\% de depresión, frecuencia similar a la encontrada en el estudio de salud mental en 2002 $(9,8 \%)(21)$.

Pocos estudios nacionales abordan la situación socio-familiar del adulto mayor; en ese sentido RuizDioses y col (22) encontraron que $75 \%$ tenía riesgo o problema social, y en nuestro estudio hemos encontrado $81,8 \%$. Se requiere de estudios que permitan valorar la situación socio-familiar de los adultos mayores y su repercusión en la salud.

Al evaluar la funcionalidad familiar a través del APGAR familiar, la disfunción familiar se presentó en 23,8\%, hallazgos similares a los encontrados por Hisako y col (23) en Portugal en 107 pacientes mayores de 80 años, encontrando disfunción familiar en $32 \%$ de su población. Esto debe alertar en la toma de medidas adecuadas ya que el ámbito familiar es el lugar de intervención anticipada en el que la percepción individual de los valores familiares podría influir en el estado de salud del adulto mayor.

Al evaluarse el riesgo de malnutrición, éste se encontró en $31,4 \%$ de los pacientes evaluados con MNA. Si bien es cierto no existe un "gold standard" para evaluar el estado nutricional en adultos mayores, el MNA se constituye en un instrumento aceptado, sensible y específico para evaluar el riesgo de malnutrición incluso antes que se refleje en el peso o en parámetros bioquímicos, por lo que se sugiere su realización periódica en algunos estudios como el de Calderón y col (24). En relación al IMC, sus valores no se encuentran definidos y estos pueden variar por diferentes causas, en nuestro estudio no se categorizó en intervalos pero sí se evaluó la presencia de obesidad con IMC > 30 encontrándose estos valores en $23,4 \%$, sin embargo cabe resaltar que dentro de los parámetros antropométricos, la circunferencia abdominal es uno de los que podrían valorar mejor la parte nutricional según algunos estudios $(25,26)$.

En relación a velocidad de la marcha como marcador de fragilidad, llama la atención que en comparación a lo encontrado por Varela y col (12) la frecuencia de frágiles es mayor duplicándose en porcentaje con una frecuencia similar en prefrágiles, si bien es cierto dicho estudio evaluó adultos mayores de la comunidad, nuestro estudio refleja una población vulnerable que requiere medidas de intervención adecuadas que permita evitar la progresión a daño y en ese sentido la velocidad de la marcha, marcador independiente de eventos adversos como caídas, fracturas, institucionalización y muerte se constituye en una prueba rápida $\mathrm{y}$ sencilla. Tal y como se reportan en varios estudios se coincide con el hecho de que la fragilidad sería más frecuente en mujeres, se incrementaría con la edad y tendría relación con factores sociales $(27,28)$.

Se considera algunas limitaciones del estudio por su diseño: en relación a los diagnósticos más frecuentes, éstos no necesariamente se consideran motivos de consulta, ya que se registraron de acuerdo a lo escrito en la historia clínica, no existiendo tampoco evidencia del uso de "gold standard" para ello; al ser un estudio descriptivo, las asociaciones encontradas deben corroborarse con otros estudios. En cuanto a las fortalezas: se usaron escalas validadas en nuestro medio para evaluar algunas variables y se constituye en el primer estudio que muestra las características de los pacientes adultos mayores que acuden a la consulta externa de Geriatría del $\mathrm{HNCH}$ lo que generará la base para la realización de otros estudios que permitan intervenir y contribuir con la mejora de la calidad de vida de los pacientes.

En conclusión, los pacientes que acuden a la consulta externa de Geriatría del $\mathrm{HNCH}$ son mayoritariamente mujeres, autónomos funcionalmente, sin deterioro cognitivo, con riesgo social y adecuada funcionalidad familiar, prefrágiles y con enfermedades crónicas no trasmisibles principalmente

\section{Correspondencia:}

Paola Casas

Jr. Nazca 367 Dpto. 404

Jesús María, Lima-Perú

Correo electrónico: paola.casas.v@upch.pe

\section{REFERENCIAS BIBLIOGRÁFICAS}

1. Instituto Nacional de Estadística e Informática. 
Perfil sociodemográfico del Perú: Censos Nacionales 2007: XI de población y VI de vivienda. Lima: INEI, UNFPA, PNUD; 2008.

2. Velásquez A. La carga de enfermedad y lesiones en el Perú y las prioridades del plan esencial de aseguramiento universal. Rev Perú Med Exp Salud Pública. 2009; 26 (2): 222-31.

3. Varela L, Sillicani A, Chávez H, Chigne O. Valoración geriátrica integral: Propuesta de addendum a historia clínica. Diagnóstico. 2000; 39 (3): 135-47.

4. Oficina de estadística e informática del Hospital Nacional Cayetano Heredia. Datos de la consulta externa 2008-2011. LIMA: Hospital Nacional Cayetano Heredia; 2012. URL disponible en: http:// www.hospitalcayetano.gob.pe/cayetanoweb/ (Fecha de acceso: mayo 2012).

5. Katz F. Use of a structures functional assessment format in a geriatric consultative service. J Am Geriat. 1988; 36: 342-437.

6. Pfeiffer E. A short portable mental status questionnaire for the assessment of organic brain deficit in elderly patient: J Am Geriat. 1975; 23: 433-41.

7. De la Torre J, Shimabukuro R, Varela L, et al. Validación de la versión reducida de la escala de depresióngeriátricaenelconsultorioexternodegeriatría del Hospital Nacional Cayetano Heredia. Acta Med Per. 2006; 23 (3): 144-47.

8. Merino R, Varela L, Manrique de Lara G. Evaluación del paciente geriátrico hospitalizado .Orientado por problemas: Estudio prospectivo de 71 casos. Rev Med Hered. 1992; 3: 51-59.

9. Good M, Smilkstein G, Good B, Shaffer T, Arons T. The family APGAR index: A study of construct validity. Journal of Family Practice. 1979; 8: 577-82.

10. Guigoz Y. Mini Nutritional Assessment: A practical assessment tool for grading the nutritional state of elderly patients. Facts and Research in Gerontology. 1994; 2:15-59.

11. Varela L, Ortiz P, Chávez H. Velocidad de la marcha como indicador de fragilidad en adultos mayores de la comunidad en Lima, Perú. Rev Esp Geriatr Gerontol. 2010; 45 (1): 22-25.

12. Gálvez M, Varela L, Chávez H, Cieza J, Mendez F. Correlación del Test "Get up and Go" con el test de Tinetti en la evaluación del riesgo de caídas en los adultos mayores. Acta Med Per. 2010; 27(1): 8-11.

13. Tello T, Varela L, Ortiz P, Chávez H, Revoredo C. Calidad del sueño, somnolencia diurna e higiene del sueño en el centro de Adultos Mayores de Mirones, Essalud, Lima Perú. Acta Med Per. 2009; 26 (1):22-26.

14. Varela L, Chávez H, Mendez F, Cerrso R, Gálvez M. INTRA III. Desarrollando respuestas integradas en sistemas de salud de una población en rápido envejecimiento. Lima: Editorial UPCH-OPS/OMS; 2005.

15. Albala C, Lebrao ML, León M, et al. Encuesta Salud, Bienestary Envejecimiento(SABE):Metodologíadela encuesta y perfil de la población estudiada. Rev Panam Salud Pública. 2005; 17 (5/6): 307-22.

16. Segura L, Agusti R, Parodi J. Factores de riesgo de las enfermedades cardiovasculares en el Perú (Estudio TORNASOL). Revista Peruana de Cardiología. 2006; 32 (2): 82-128.

17. Huicho L, Trelles M, Gonzales F, Mendoza W, Miranda J. Mortality profiles in a country facing epidemiological transition: An analysis of registered data. BMC public health. 2009; 9:47.

18. Secoli R. Polifarmacia: Interacciones y reacciones adversas en uso de medicamentos por ancianos. Rev Bras Enferm. 2010; 63 (1): 136-140.

19. Ocampo JM, Chacón A, Gómez JF, Curcio CL, Tamayo F. Reacciones y eventos adversos por medicamentos en ancianos que consultan un servicio de urgencias. Colomb Med. 2008; 39: 135-46.

20. Pinheira MA, Facchini L, Thumé E, Maia F. Gender and incidence of functional disability in the elderly: A sistematic review. Cad Saúde Pública. 2009; 25 (S3): S464-76.

21. Instituto Nacional de Salud Mental Honorio DelgadoHideyo Noguchi. Estudio Epidemiológico de la Salud Mental en Fronteras 2005. Informe general. Anales de Salud Mental. 2006; 22 (1-2): 5-223.

22. Ruiz-Dioses L. Situación Sociofamiliar, valoración funcional y enfermedades prevalentes del adulto mayor que acude a establecimientos de I nivel de atención, Callao 2006. Rev Peru Med Exp Salud Pública. 2008; 25(4): 354-79.

23. Hisako L, Mendez M, Martins M, Nassar S. La dinámica de la familia de ancianos con edad avanzada en el contexto de la ciudad de Porto, Portugal. Rev Latin-Am Enfermagem. 2011; 19 (3): 1-9.

24. Calderon M. Evaluación Nutricional comparada del adulto mayor en consultas de Medicina Familiar. Nutr Hosp. 2010; 25 (4): 669-75.

25. Perissinotto E. Anthropometric measurements in the elderly: age and gender differences. Br J Nutr. 2002; 87: 177-86.

26. Woo J, Yu R, Yau F. Fitness, fatness and survival in the elderly populations. Age (Dordr). 2012 Mar 7. [Epubahead of print]. URL disponible en: http:// www.springerlink.com/content/686nv35351044v8q/ ?MUD=MP (Fecha de acceso: mayo 2012).

27. Nasiya A, Mandel R, Fain M. Frailty: An emerging geriatric syndrome. Am J Med. 2007; 120 (9): 74853.

28. Fried LP, Tangem CM, Walston J. Frailty in older adults: evidence for a phenotype. J Gerontol A Biol Sci Med Sci. 2001; 56:146-56.

Recibido: 01/06/12

Aceptado para publicación: 19/11/12 